\title{
Augmented Quick Health
}

\author{
S.M.A. Sharif ${ }^{1}$, Iftekharul Mobin ${ }^{2}$, and Nabeel Mohammed ${ }^{3}$

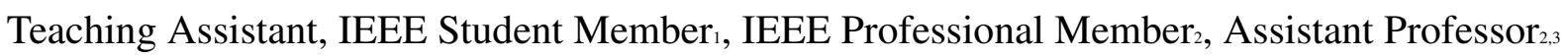 \\ Department of Computer Science and Engineering \\ University of Liberal Arts Bangladesh, 4/A Dhanmondi, Dhaka- 1209
}

\begin{abstract}
Personalized Health Assistants have gained popularity over the last few years. Such technologies allow users to monitor their health information in real time and often integrate with their smart devices, especially smart phones. Augmented Quick Health $(\mathrm{AQH})$ is such an intelligent health monitoring system, which uses multiple sensors to read heartbeat, body temperature and sweat rate information. The sensor readings are used together to determine the health condition of a subject. With the resulting output, it interacts with a smart device and data is pushed in to a robust cloud-based infrastructure via the device (e.g. smartphone). The system is designed to be extensible and flexible, so adding new sensors and/or use-cases is straightforward. While there are other personalized health monitoring systems, AQH incorporates location based search, presented using augmented reality, which has proven to be an effective tool in emergency situations.
\end{abstract}

\section{General Terms}

Health Monitoring, Emergency Help Search

\section{Keywords}

Wearable Device, Real time health monitoring, Augmented Reality, Cloud Server, Rest Application, Augmented Quick Health(AQH)

\section{INTRODUCTION}

Health related devices and applications are promising research area of IoT [1] and blessed by the advancement of new era of information communication technology. The true potential of health application system lies in providing real time physical information to individual. Moreover, the system can collect data from monitored individual person and accumulated data can be used to establish a 24/7 information bridge between health service consumers and service providers such as: doctors, consulting centers, diagnostic centers, clinics, hospitals, therapists, pharmacists etc. Patient monitoring, medication, diagnosis and health related various services can be improved enormously [2]. This paper proposing such a system that will have a linkup between smartphone and real time health monitoring. Our system is concern with making health monitoring easy, let physician or family member know about health status update in real time and also can find out location base emergency help station by the use of smartphone internet connection to rescue patient in the minimum time or from the shortest distance. The Augmented Quick Health (AQH) is an intelligent system assembled with multiple sensors and operated by micro-controller unit (MCU) to monitor human health status in real time. From the connected sensors embedded on the body, it takes reading of heartbeat, body temperature and sweating. According to pre-defined logic and from resulting output, it determines the health condition. Similar approaches are published before. But this system is also incorporated with location based search facility for emergency help.Proposed system will make health monitoring more efficient, user friendly and low in cost.Finding of the study is that,this system demonstrated its efficiency most of the cases perfectly.

\section{BACKGROUND OF THE STUDY}

Embedded system based on health monitoring is an emerging field for research. There are some health care device and applications are available at present [8, 3] and some researchers [6] are still working on the improvement of existing system. Many of them worked with the wearable devices [5]. For an instance, Chan, Selvaraj and Ferdosi [5] conducted their research on wireless patch sensor for remote monitoring of heart rate, respiration, activity, and falls. There are also some other groups of people who tried to monitor older people by using sensors and smart phone (i.e. Fook [6]). Simulation results are shown for the android device for their prototype. Another health related project U-Health has been proposed in [9] for monitoring user health status and send user health related data to the physician. However, still there are extensive research challenges and issues that needs to overcome [10]. One of the major challenges which are overlooked previously by many researchers was actual device implementation according to proposed prototype [4, 5, 6]. Main focus of this paper to provide every user a complete system that provide users facility of monitoring their health status in real time and also provide them the facility of searching emergency help and also a chance to monitor other user's health status in real time as well. For the experiment, a prototype of wearable device has been introduced such a way that it can demonstrate all features and can be deploy in AQH system comfortably. To making this prototype, only those sensors have been used that are related with the smartphone app features. Since normal value of heart beat is depending on age and gender, different algorithm for different age and genders have been used. Moreover, traditional abnormali- 
ties detection approach temperature measurement is included as it indicates most common human health condition [11] 12].

\section{SYSTEM DESCRIPTION}

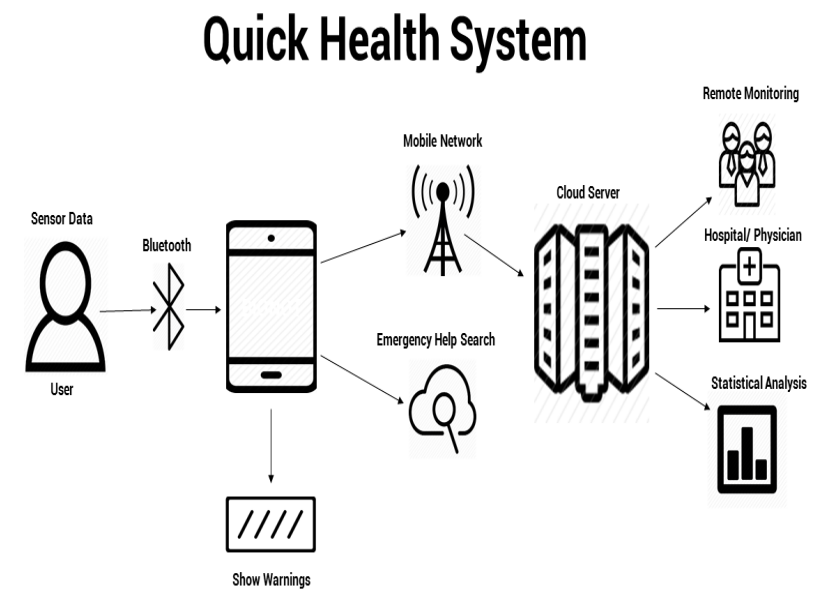

Fig. 1: QH System Overview

Augmented Quick Health is designed such a way that, it can monitor one's health status, analyze physical condition, provide warnings and advises user what to do according to health condition in an emergency situation. It can search emergency help and redirect user to the nearest hospitals. Quick Health consists of three basic parts.

\section{(1) Wearable Device \\ (2) BIONOT (Smartphone Application) \\ (3) Cloud Server}

Fundamental work of wireless device is to monitoring user's body condition and feed these data to the smart-phone application via Bluetooth connection. BIONOT (smartphone application) will receives data from the device and begins to analyze all data by using predefined logic. According to analysis the smartphone application determines the physical condition of the user. Whenever it detects the collected values have crossed the normal limits, BIONOT produces warnings and also provides advises and recommendations. There is a unique feature of Quick Health system. This exclusive feature is whenever any user needs any medical help, user can search emergency help in two away. First approach of searching emergency help search is send a message to the predefined contact numbers and another approach is search the nearest hospitals by the use of augmented reality concept[16]. This smartphone application is also responsible to connect cloud server and store user data in the cloud storage for the further analysis. Beside this Quick health is also provide a feature of real time remote monitoring of other users from the application based on shared permission from other users of QH and BIONOT. But to do so user must need allow other users and select from the server to whom he/she is willing to disclose and share health information or status. A relevant data flow diagram is shown in the figure 2 .

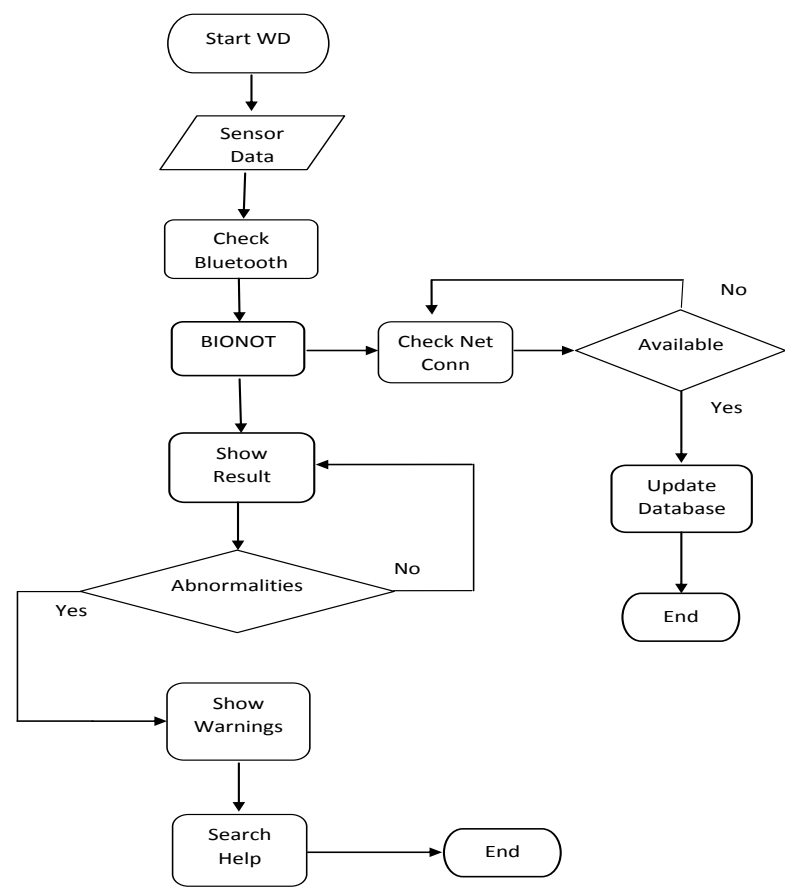

Fig. 2: QH System Overview

\subsection{Wearable Device}

We have prepared a prototype of wearable device with the Arduino and required sensor modules. This wireless device takes reading from different portion of human body and pass all data to the smart phone application by the using of Bluetooth connectivity. The circuit diagram of wearable device is shown in figure 1. For determining health condition we have taken three classical body parameter and they are 1. Heartbeat 2. Body Temperature and 3. Sweating. Two arduino compatible sensors have been used for sensing this parameters. We have used Grove Ear-clip Heart Rate Sensor model number MED03212P [13] for heart beat measurement and Temperature and humidity sensor model number SEN11301P [14] for Heat and Sweating Measurement (Figure 4)in our prototype (see figure 4). There is also a push button has been used for producing a emergency signal. When user will press this button, the mobile phone application will generates and send an emergency massage to particular correspondence with user current body status and location. Behavior of sensors are tracked in the several curves and discussed in the section 5. All collected data will send to the smartphone application through a Bluetooth module. HC-05 [15] module have been used for setup and sending data from arduino. Circuit diagram of device is shown in the figure 3 .

\subsection{Smartphone Application(BIONOT)}

It is the vital and key element of AQH. We have developed it in the android platform. This mobile app will works as manager of the device as well as showing all information, statistical data, warning and so on. It also generates help message and redirect user to the correct physician and locate user current location. It will also sends user data to the cloud server with the permission of user. The BIONOT application contains lots of activities and pages. At the very beginning user have to register with his name, age, gender and have to provide his emergency contact. On the next interface he 

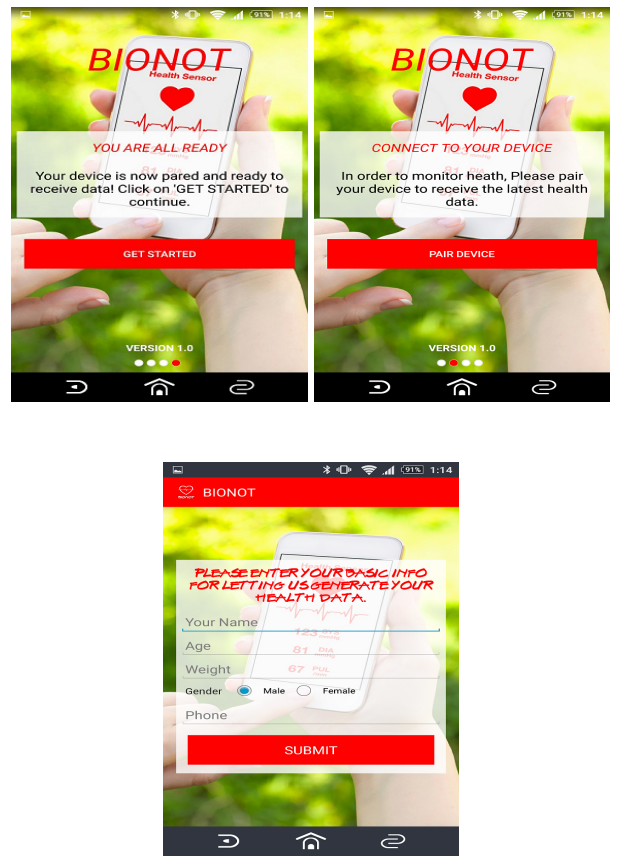

Fig. 4: Startup, login and configuration of smart phone BIONOT apps

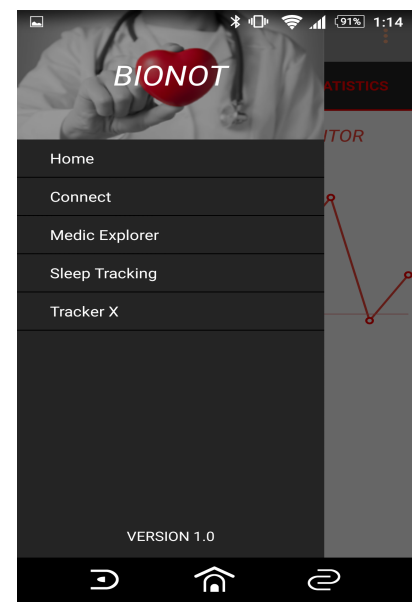

Fig. 5: Quick Menu of smart phone BIONOT apps

would select and pair his device with the application via Bluetooth with wearable device prototype. As it got paired it will start to receive data from the device. Some screen shot from BIONOT are shown in the figure 4-7.

3.2.1 Dashboard (figure 6). The Dashboard (home screen) is dedicated for monitoring overall health condition include heartbeat, body temperature and current sweating condition. There is a heart sign in the screen that will pop up according to the heartbeat rate of sample.

3.2.2 Heart Menu (figure 7). The second screen is dedicated to heart beat monitoring. Which we have been used for checking current heartbeat and the average of his heart beat rate.

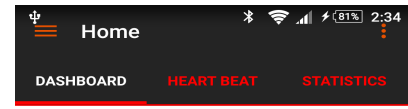

HEALTH STATUS IS NORMAL

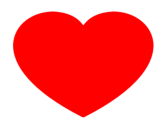

$37_{\text {CELSIUS }}^{\text {TEMPRAT }}$

87 PERATING

84 HEARTBEAT

\section{๑ $\curvearrowright$}

Fig. 6: Dashboard of mobile application in smart phone

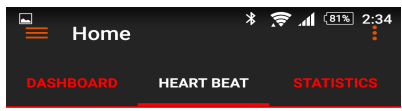

REALTIME HEARTBEAT MONITOR
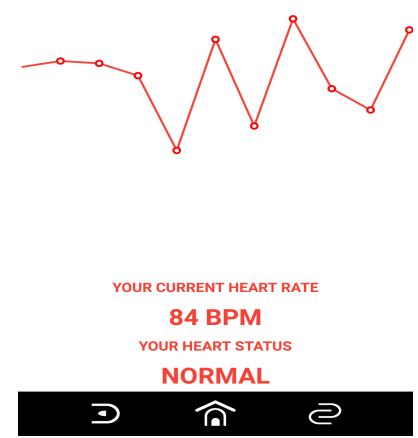

Fig. 7: Heart Menu of smart phone BIONOT apps

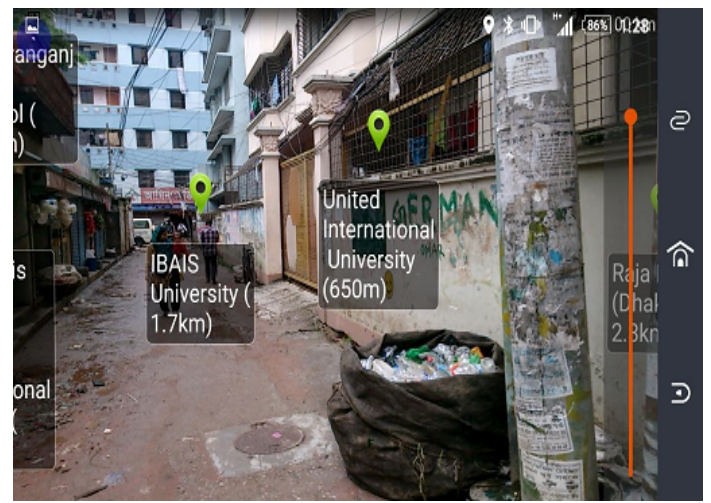

Fig. 8: Medic Explorer is an unique feature of Quick Health

\subsection{Cloud Server}

Every user will be connected to the cloud server after the successful completion of the registration process. There is also a option for which user can able to select who will can monitor their health 
related data by using of tracker-x, thus privacy also will be maintained. User can also send their body statistics directly to the physician through our data server. A complete overview of Quick Health is given in flow chart figure that already shown in the figure 3 . In our server side, we have wrote a RESTful application. This application will receive user data from the smartphone application and store all data in the Quick Health central database. In our REST application there are specific api's for all individual function. Which allow smartphone application to connect and retrieve every individual data for the users.

\section{KEY FEATURE OF QH (EMERGENCY HELP SEARCH)}

Quick Health is not about only real time health status monitoring. It also concerned with rescue user in critical health condition. We have integrated two different types of help facilities in our system. One is from the wearable device. another one is from the smartphone application.

\subsection{One click Emergency Help Search}

In the prototype of wearable device we have integrated a push button, which allow user to search help from their predefined contact numbers. When user will press this button the MCU(arduino) will send a signal to the smartphone application through Bluetooth connectivity.

\subsection{Medic Explorer}

This is the most impotent feature of quick health system. By the use of magnetic sensor of smart-phone it determines the direction and then searches for the hospitals or medical centers. Whenever it discovers any hospital or medical center in the map, it will find out the nearest one from the current location among all discovered hospitals. Finally, it shows the distance and area in the display and redirect user to the nearest medical center. We have implemented the concept of augmented reality in Medic Explorer. Figure 8 is a sample snapshot of augmented reality based location search.

\section{RESULTS AND DISCUSSION}

Data of used sensor (mentioned above) analyzed in that section . Data is taken from the Arduino in an interval of 20 seconds. According to fetched data and the shown curves showed that wearable device prototype is able to produce the continuous data and most of the data are relevant comparing a normal human health condition. Behavior of the different sensor used in the prototype are discussed in the section 5.1-5.2.

\subsection{Heart Beat Measurement}

The prototype of wearable vevice has tested on a person of age 24. Amused that, the person's health was in stable condition. The curve of 100 sample discussed in that section shows that our sensor is performing quit nicely (shown in the figure 9). its able to take continues values from the persons body and most of them are relevant.

\subsection{Sweating and Body temperature Measurement}

A all sensors are integrated in the same device, so that this sensor is also tested on the same person. As it was tested in the mid of June' 15 and that was the summer season in the Bangladesh, the person was sweating and temperature is also high. Figure 10-11

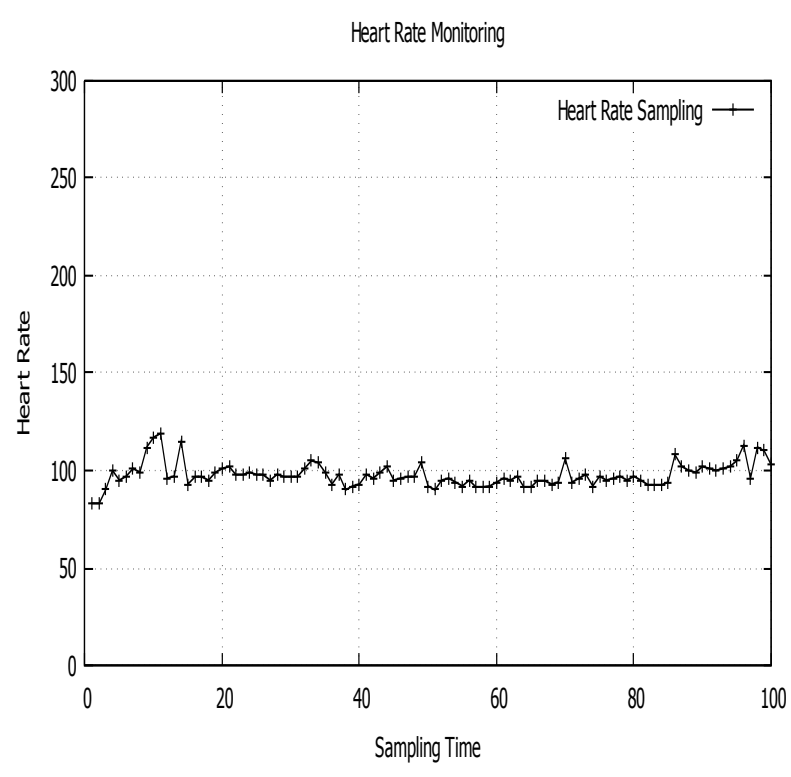

Fig. 9: Heart Beat sensor

showing the same result. Most of the values of figure 10-11 are relevant and acceptable.

\section{FUTURE SCOPE}

Some others feature like calorie burn, sleep detection, movement and distance covered etc can be detected by the use of other sensors. We are planning to add those features in future within wearable device and will integrate all those data in cloud storage. Automatic analysis will be added for auto prescription and recommendation generation. We are willing to add all those parameter with our device and can find out the co-relation between various parameters effect on human health. Our future aim of us, to work with heart patients data that will facilitate us to compare the health condition, and device reliability more accurately.

\section{CONCLUSION}

The topics covered in this paper are implemented successfully. Most of the cases the system behave quit nicely as predefined logic. Prototype of the wearable device is also has successfully synced with the android platform based smartphone application. Our smartphone application also able to connect our cloud server and it could show the all possible help station from the map according to user location. $\mathrm{AQH}$ is a simple solution for real time health status monitoring and emergency help searching. This system is also very user friendly. It has been deployed such a way that, user can use it without any knowledge about the system. Augmented reality made help searching more easier comparing to the existing help searching techniques. Moreover, AQH is a complete solution for real time health care monitoring and emergency help search facility.

\section{REFERENCES}

[1] V. Turner, J. F. Gantz, D. Reinsel, and S. Minton, "The digital universe of opportunities: Rich data and the increasing 


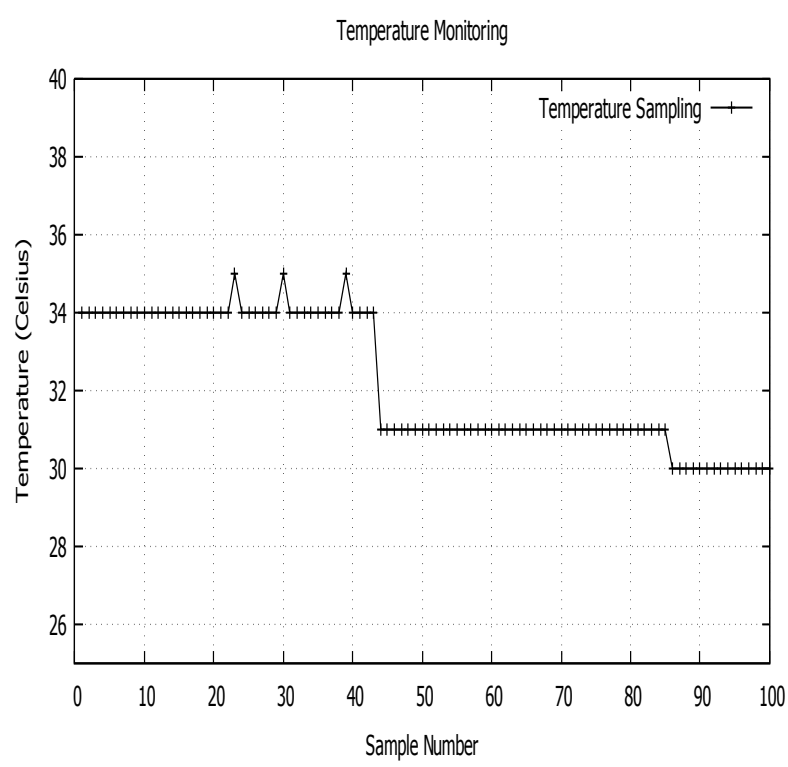

Fig. 10: Statistics curve of body temperature for 100 samples

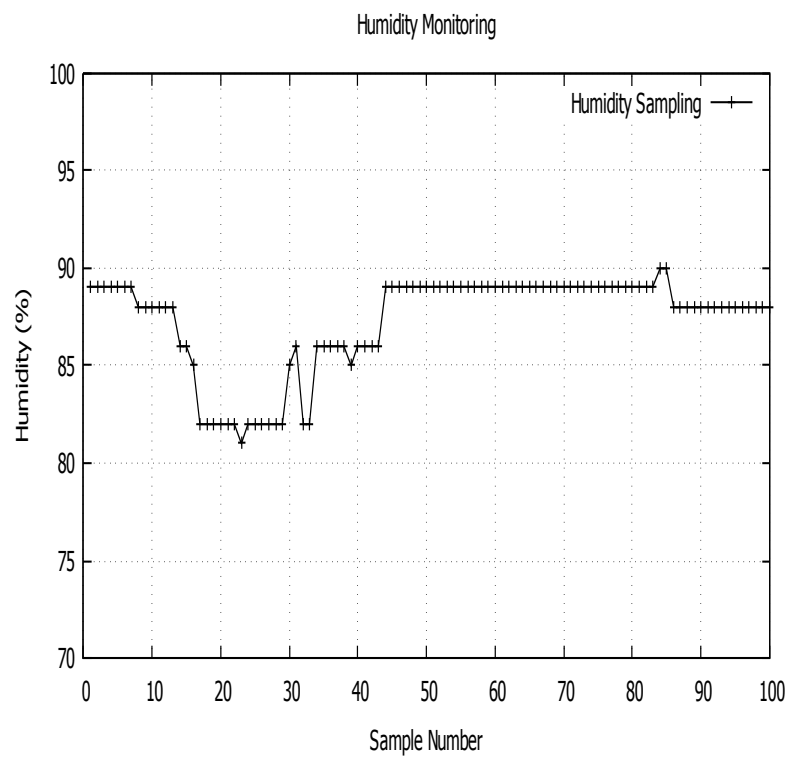

Fig. 11: Statistics curve of body sweating for 100 samples

value of the internet of things," International Data Corporation, White Paper, IDC_1672, 2014.

[2] A. D. Black, J. Car, C. Pagliari, C. Anandan, K. Cresswell, T. Bokun, B. McKinstry, R. Procter, A. Majeed, A. Sheikh et al., "The impact of ehealth on the quality and safety of health care: a systematic overview," PLoS medicine, vol. 8, no. 1, p. 188, 2011.

[3] G. Suciu, V. Suciu, S. Halunga, and O. Fratu, "Big data, internet of things and cloud convergence for e-health applications," in New Contributions in Information Systems and Technologies. Springer, 2015, pp. 151-160.
[4] D. M. Zulman, E. C. Jenchura, D. M. Cohen, E. T. Lewis, T. K. Houston, and S. M. Asch, "How can ehealth technology address challenges related to multimorbidity? perspectives from patients with multiple chronic conditions," Journal of general internal medicine, pp. 1-8, 2015.

[5] A. M. Chan, N. Selvaraj, N. Ferdosi, and R. Narasimhan, "Wireless patch sensor for remote monitoring of heart rate, respiration, activity, and falls," in Engineering in Medicine and Biology Society (EMBC), 2013 35th Annual International Conference of the IEEE. IEEE, 2013, pp. 6115-6118.

[6] M. Clark, J. Lim, G. Tewolde, and J. Kwon, "Affordable remote health monitoring system for the elderly using smart mobile device," Sensors \& Transducers (1726-5479), vol. 184 , no. 1,2015 .

[7] J. Carmigniani, B. Furht, M. Anisetti, P. Ceravolo, E. Damiani, and M. Ivkovic, "Augmented reality technologies, systems and applications," Multimedia Tools and Applications, vol. 51, no. 1, pp. 341-377, 2011.

[8] P. Castillejo, J.-F. Martinez, J. Rodríguez-Molina, and A. Cuerva, "Integration of wearable devices in a wireless sensor network for an e-health application," Wireless Communications, IEEE, vol. 20, no. 4, pp. 38-49, 2013.

[9] Y. E. Gelogo and H.-K. Kim, "Integration of wearable monitoring device and android smartphone apps for u-healthcare monitoring system." International Journal of Software Engineering \& Its Applications, vol. 9, no. 4, 2015.

[10] D. K. Ahern, "Challenges and opportunities of ehealth research," American journal of preventive medicine, vol. 32, no. 5, pp. S75-S82, 2007.

[11] "Heartrate algorithm," http://www.heart.org/HEARTORG/ GettingHealthy/PhysicalActivity/FitnessBasics/ Target-Heart-Rates_UCM_434341_Article.jsp accessed On: 2015-06-26.

[12] K. Christensson, T. Cabrera, E. Christensson, K. UvnäsMoberg, and J. Winberg, "Separation distress call in the human neonate in the absence of maternal body contact," Acta paediatrica, vol. 84, no. 5, pp. 468-473, 1995.

[13] "Grove Ear clip heart Beat Sensor"[Online]. Available: http://www.seeedstudio.com/wiki/Grove_-_Heart_rate_ear_ clip_kitp [Accessed: 19-12-2015]

[14] "Temperature and Humidity Sensor"[Online]. Available: http://www.seeedstudio.com/wiki/Grove_-_Temperature_ Sensor/[Accessed: 19-12-2015]

[15] "HC-05," [Online]. Available http://www.electronica60norte. com/mwfls/pdf/newBluetooth.pdf [Accessed: 19-12-2015]

[16] "Augmented Reality,"[Online]. Available http://mashable. com/category/augmented-reality/[Accessed: 19-12-2015] 


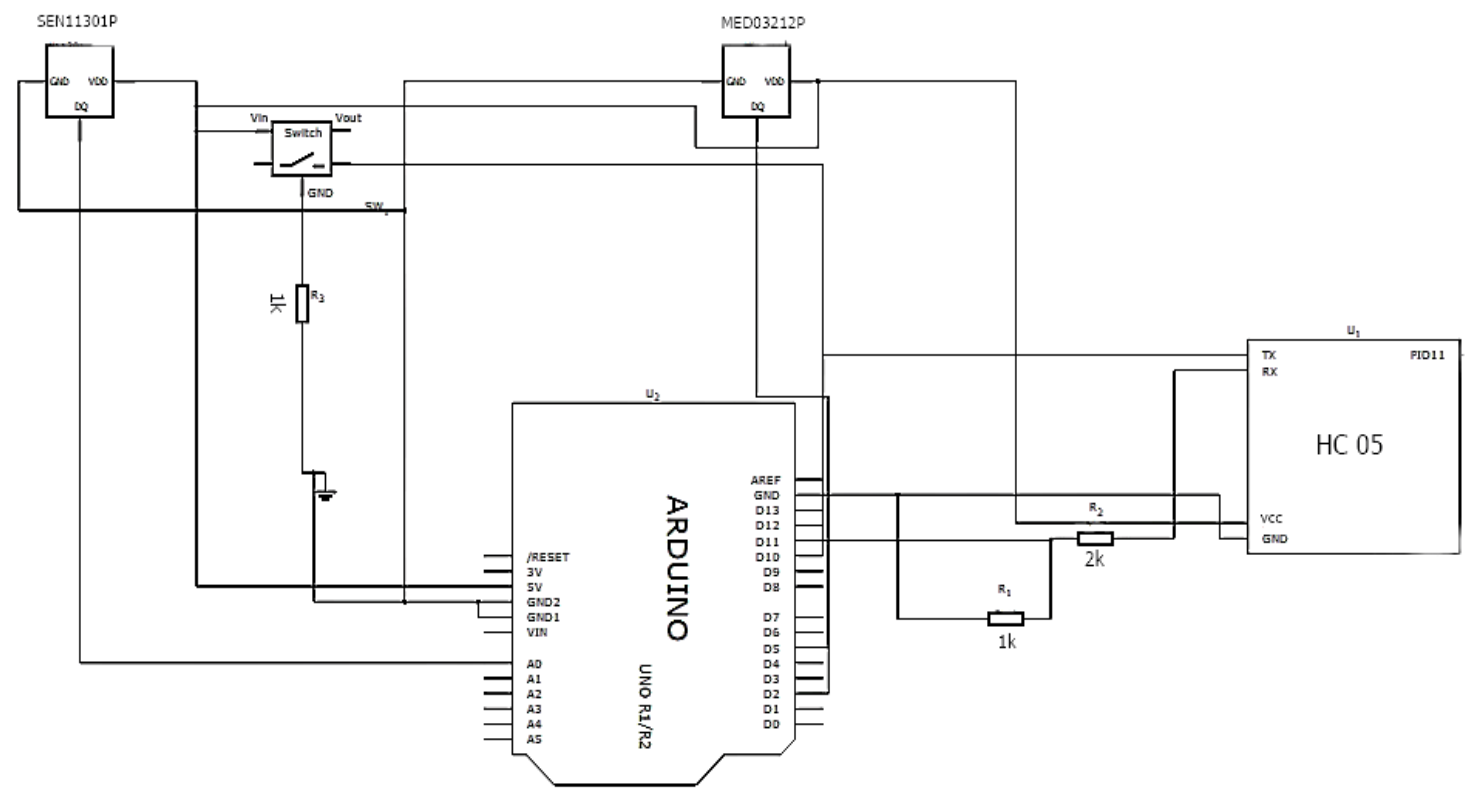

Fig. 3: Circuit Diagram of bodycentric QH wireless device 